\title{
Ruptured pseudoaneurysm of the splenic artery complicating endoscopic ultrasound-guided fine- needle aspiration biopsy for pancreatic cancer
}

Endoscopic ultrasound-guided fine-needle aspiration (EUS-FNA) is a safe procedure, although major complications do rarely occur. Pseudoaneurysm rupture is an emergency that can cause life-threatening hemorrhage. An exceedingly rare case of ruptured pseudoaneurysm of the splenic artery following EUS-FNA is described.

A 62-year-old man was referred for investigation of a pancreatic tumor. On abdominal computed tomography (CT), a lowdensity area was seen running from the pancreatic body, involving the celiac and splenic arteries ( Fig. 1). Angiography showed encasement of the splenic artery ( $\bullet$ Fig. 2). Therefore, EUS-FNA was performed with two separate passes into the lesion using a 22-gauge needle. Cytological analysis showed a pancreatic adenocarcinoma. Nineteen days later abdominal CT showed a pseudocyst in the pancreatic body and tail ( Fig. 3), and 30 days later (i.e., 30 days after EUS-FNA) the patient developed hematemesis and hemorrhagic shock. Upper gastrointestinal endoscopy showed a hemorrhagic gastric ulcer in the posterior wall of the middle body $(\bullet$ Fig. 4). Since endoscopic treatment was unsuccessful, angiography was performed and showed a pseudoaneurysm of the splenic artery. Coil embolization was performed in and around the pseudoaneurysm. After 7 days the pancreatic pseudocyst had narrowed ( $\bullet$ Fig. 5); there was no further recurrence.

The overall complication rate of EUS-FNA is $1 \%-2 \%$ [1]. The major complications are postaspiration infection in cystic lesions, bleeding, pancreatitis, and cervical and duodenal perforation [2]. Three different mechanisms are recognized as causing pseudoaneurysm formation [3], but all are called pseudoaneurysm because the end result is a cystic vascular structure surrounded by a fibrous wall. Pseudoaneurysms can rupture into the peritoneal cavity, retroperitoneum, gastrointestinal tract, biliary tract, and pancreatic duct. The most common vessel involved is the splenic artery as it runs along the pancreatic bed before reaching the spleen [4]. To our knowledge, this is the

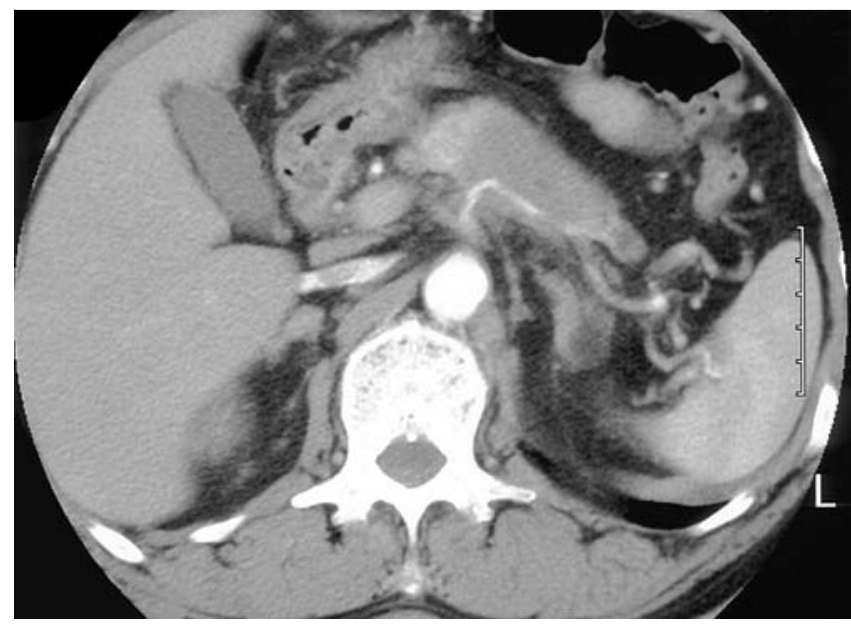

Fig. 1 On abdominal $\mathrm{CT}$, a low-density area was seen running from the pancreatic body, involving the celiac and splenic arteries.

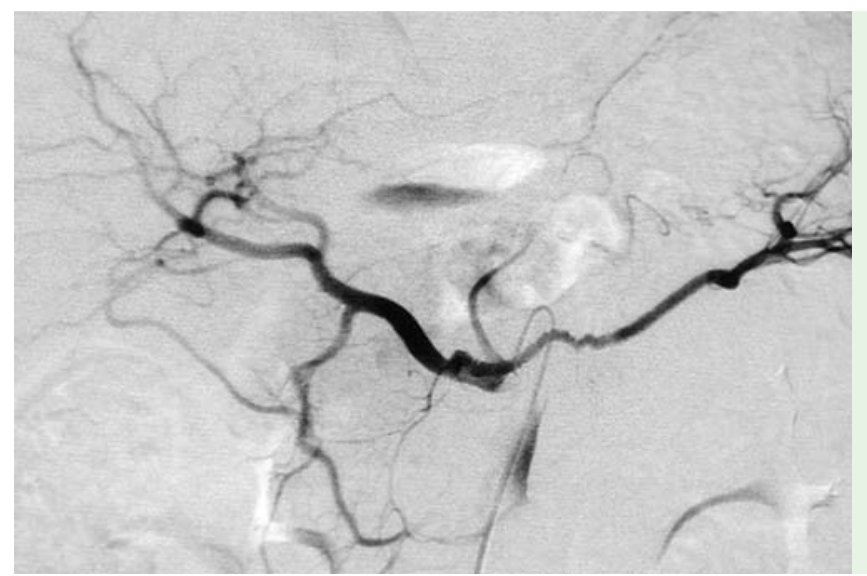

Fig. 2 Angiography showed encasement of the splenic artery.

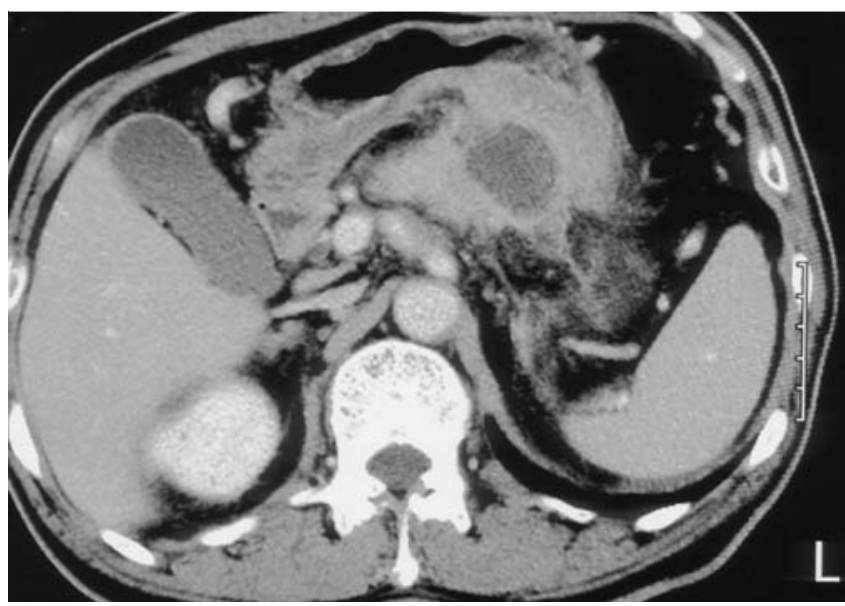

Fig. 3 Abdominal CT 19 days later showed a pseudocyst in the pancreatic body and tail. 


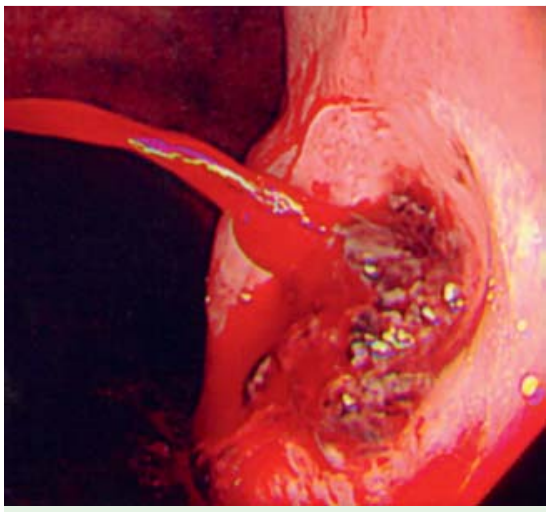

Fig. 4 Upper gastrointestinal endoscopy showed a hemorrhagic gastric ulcer in the posterior wall of the middle body.

first case report of a ruptured pseudoaneurysm of the splenic artery complicating EUS-FNA.

\section{Endoscopy_UCTN_Code_CPL_1AL_2AD}

\section{K. Matsumoto ${ }^{1}$, K. Hara ${ }^{1}$, A. Sawaki ${ }^{1}$,} N. Mizuno ${ }^{1}$, S. Hijioka ${ }^{1}$, H. Imamura ${ }^{1}$, Y. Niwa², M. Tajika², H. Kawai²,

S. Kondo ${ }^{2}$, Y. Inaba ${ }^{3}$, K. Yamao ${ }^{1}$

1 Department of Gastroenterology, Aichi Cancer Center Hospital, 1-1 Kanokoden, Chikusa-ku, Nagoya 464-8681, Japan

2 Department of Endoscopy, Aichi Cancer Center Hospital, Nagoya, Japan

3 Department of Diagnostic and Interventional Radiology, Aichi Cancer Center Hospital, Nagoya, Japan

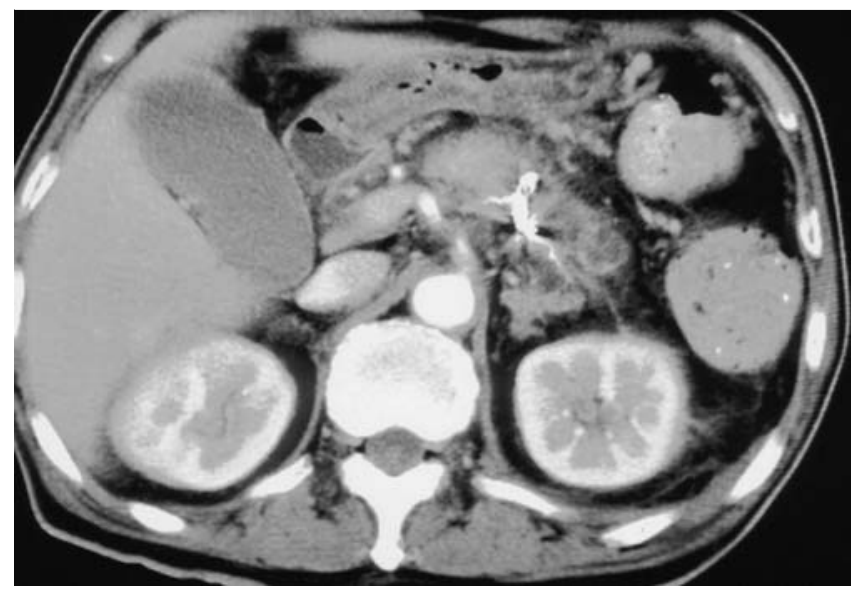

Fig. 5 Coil embolization in and around the pseudoaneurysm was performed, and 7 days later the pancreatic pseudocyst had narrowed.

\section{References}

1 Bhutani MS. Endoscopic ultrasound guided fine needle aspiration of pancreas. In: Bhutani MS (ed). Interventional endoscopic ultrasonography. Amsterdam: Harwood Academic, 1999: 65-72

2 O'Toole D, Palazzo L, Arotcarena $R$ et al. Assessment of complications of EUS-guided fine-needle aspiration. Gastrointest Endosc 2001; 53: 470-474

3 Hsu JT, Yeh CN, Hung CF et al. Management and outcome of bleeding pseudoaneurysm associated with chronic pancreatitis. BMC Gastroenterol 2006; 6: 3

4 Tessier DJ, Stone WM, Fowl RJ et al. Clinical features and management of splenic artery pseudoaneurysm: case series and cumulative review of literature. J Vasc Surg 2003; 38: $969-974$

\section{Bibliography}

DOI $10.1055 / \mathrm{s}-0029-1215323$

Endoscopy 2010; 42: E27-E28

(c) Georg Thieme Verlag KG Stuttgart · New York · ISSN 0013-726X

\section{Corresponding author}

\section{K. Yamao, MD}

Department of Gastroenterology

Aichi Cancer Center Hospital

1-1 Kanokoden

Chikusa-ku

Nagoya 464-8681

Japan

Fax: +81-52-7642942

kyamao@aichi-cc.jp 